\title{
Regulación de las nanotecnologías en América Latina
}

William Alonso Urquijo*

La regulación de las nanotecnologías está aún en pañales a nivel mundial, no obstante

que los productos entran al mercado día a día con riesgos imprevisibles a la salud y el medio ambiente. Europa tímidamente comenzó a etiquetar algunos productos, pero la discusión está en auge a nivel mundial. En América Latina, México ya ha emitido unos

lineamientos de regulación que, aunque voluntarios, constituyen una advertencia de que se inclinará a favor de los criterios de Estados Unidos. Brasil ha agendado reuniones ministeriales para tratar el tema. El lector puede obtener una puesta al día sobre la regulación de las nanotecnologías en este breve artículo.

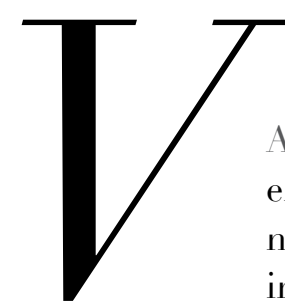

ARIAS INSTITUCIONES trabajan en la formulación de reglamentaciones para los nanomateriales a nivel internacional y nacional. En algunos de estos organismos participan países de América Latina, como en el Strategic Approach to International Chemicals Management (SAICM), la Organización para la Cooperación y el Desarrollo Económicos (OCDE), la International Organization for Standardization (ISO) y otras. Además, los países latinoamericanos están atentos a las regulaciones que presentan Europa y Estados Unidos.

* Abogado experto en Nuevas Tecnologías. Miembro de la Red Colombiana de Nanociencia y Nanotecnología, Colombia (www. nanoderecho.com). Correo electrónico: urquijog@hotmail.com
SAICMI $^{1}$ es una plataforma internacional multilateral y no vinculante que tiene como propósito la sustentabilidad del manejo de químicos. Las acciones de SAICM son resultado de los acuerdos por consenso de la International Conference on Chemicals Management (ICCM). La Tercera Conferencia Internacional de la ICCM fue realizada en 20I2. Allí se adoptó la resolución III/2 sobre cuestiones emergentes de política, dentro de las cuales se incluyó a las nanotecnologías y nanomateriales manufacturados. ${ }^{2}$ Aunque los acuerdos no son vinculantes, esto representa un compromiso de los gobiernos firmantes, entre los cuales se encuentran casi todos los de América Latina. La conferencia decidió incluir los nanomateriales al Plan Global de Acción de SAICM, lo cual significa que los países se 
comprometen a asumir la discusión pública sobre políticas, transparencia y divulgación de las implicaciones de las nanotecnologías, la posibilidad de generar registros o inventarios de actividades de mercado de los nanomateriales, la promoción de disponibilidad de información sobre la presencia de dichos nanomateriales tanto dentro de la cadena de valor como a lo largo del ciclo de vida, esto puede incluir el etiquetado de acuerdo con requerimientos y guías internacionales; también se acordó invitar a los expertos en transporte de productos peligrosos y del sistema global de armonización, clasificación y etiquetado de químicos (Globally Harmonized System of Classification and Labeling of Chemicals -GHS-) a revisar la aplicación del mismo a los nanomateriales manufacturados. ${ }^{3}$ Esto abre un espacio político importante a nivel nacional y regional para la participación de los diversos agentes interesados en la regulación de las nanotecnologías.

La Unión Europea es la región más avanzada en términos de reglamentación de las nanotecnologías, y los países de América Latina también están atentos a lo que allí ocurre. Con el documento "Hacia una estrategia europea para las nanotecnologías" de 2004, la Unión Europea comienza institucionalmente el debate para un marco regulatorio en el tema. ${ }^{4}$ En 20 oI define los nanomateriales como: "A natural, incidental or manufactured material containing particles, in an unbound state or as an aggregate or as an agglomerate and where, for $50 \%$ or more of the particles in the number size distribution, one or more external dimensions is in the size range I $\mathrm{nm}$ - Ioo $\mathrm{nm} .{ }^{5}$

Esta definición contiene algunas cuestiones que se deben resaltar: 1) se incluye tanto a los nanomateriales manufacturados como a los naturales y los que resultan fortuitos de los procesos de producción. Esto es importante porque documentos de Estados Unidos, por ejemplo, sólo reconocen en su política a los nanomateriales que son intencionalmente producidos, dejando de lado los naturales y fortuitos;2) se marca un mínimo de 50 por ciento o más de las partículas con al menos una dimensión nano. Puede haber materiales con menos de 50 por ciento de nanopartículas que cumplan funciones nuevas, y que no serían catalogados como nanomateriales por razón de esta definición, y 3) se define la nanopartícula por el tamaño I a roo nm, y no por desarrollar funciones nuevas, como sí lo hace, por ejemplo, la definición de la OCDE. Hay materiales que con 3 oo nanómetros manifiestan propiedades nuevas que no estarían inclui- das según la definición europea. La definición de nanomateriales es aún objeto de controversia internacional.

La principal legislación europea para nanomateriales se llama Registro, Evaluación, Autorización y Restricción de Sustancias Químicas (REACH, por sus siglas en inglés) y regula los productos químicos. Esto presenta un problema, ya que REACH no fue elaborada para nanomateriales, por lo cual los criterios toxicológicos no se ajustan a las propiedades nanométricas.

No obstante, la Unión Europea es la región más avanzada en materia de regulación de nanotecnologías. A la fecha ha implementado lo siguiente:

I. Cosméticos. Regulación (EC I223/2009). Requiere presentación del producto con seis meses de antelación a su lanzamiento al mercado, con lista de ingredientes y características toxicológicas y de exposición. El producto debe ser etiquetado, incluyendo ingredientes.

II. Alimentos. Regulación (EU No. II69/20II). Semejante a cosméticos.

III. Biocidas. Regulación (EU) 528/2012. El producto requiere autorización previa, mayor especificación que en los casos anteriores y también etiquetado.

Para los países de América Latina que tienen acuerdos de libre comercio con Estados Unidos, la política de éste en materia de nanotecnología es clave. En Estados Unidos muchos nanomateriales pueden entrar en el Toxic Substance Control Act (TSCA) que reclama para las nuevas sustancias químicas la aprobación pre-mercado. Allí han entrado, por ejemplo, varios tipos de nanotubos de carbono, nano-sílice y nano-alúmina. Pero en el caso de Estados Unidos, a diferencia del europeo, es la agencia encargada - en este caso Environmental Protection Agency's (EPA) - quien debe demostrar que el producto manifiesta importantes riesgos (unreasonable risks), en lugar de ser el mismo productor como en el REACH de la Unión Europea. Aunque no está escrito, el concepto de unreasonable risk que se utiliza en Estados Unidos consiste en que los riesgos sean mayores al beneficio económico, con lo que se pueda entender por ello ("The legislative history, however, indicates that unreasonable risk involves the balancing of the probability that harm will occur and the magnitude and severity of that harm against the effect of a proposed regulatory action on the 


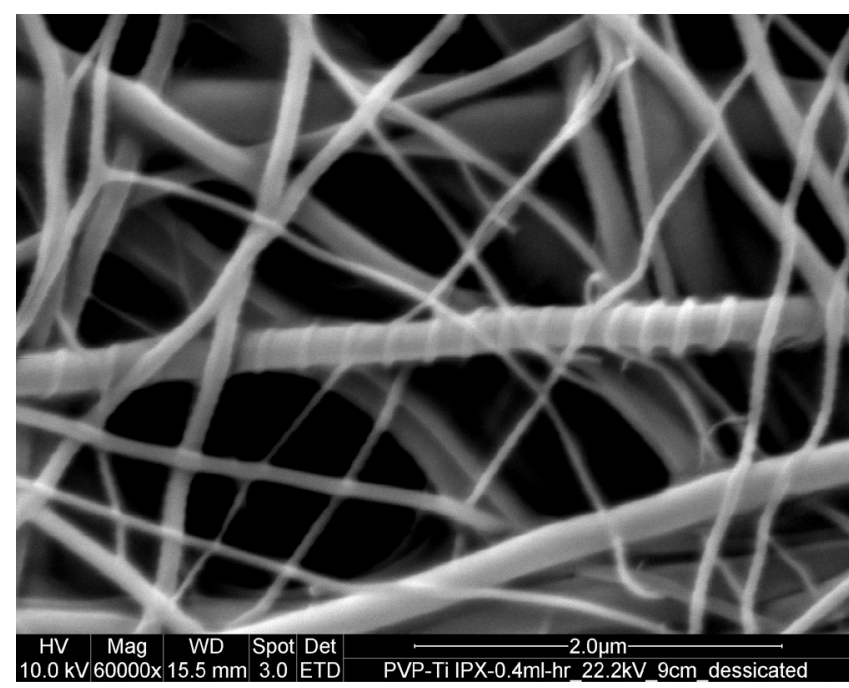

availability to society of the expected benefits of the chemical substance"). ${ }^{6}$ De no haber respuesta por parte de la EPA en go días, el producto automáticamente es aprobado para entrar al mercado. Además de la reglamentación del TSCA, que afecta y puede afectar nanopartículas, Estados Unidos tiene unos lineamientos voluntarios elaborados por el Departamento de Comercio y otros, con los cuales busca presionar a sus socios comerciales para que los adopten y se armonicen los criterios comerciales. Estos lineamientos están más centrados en solucionar potenciales trabas comerciales que en promover la seguridad de los productos.7 México, como veremos, ya ha aceptado una versión muy semejante de estos lineamientos a nivel interno.

De los países de América Latina, México y Brasil están dando sus primeros pasos en la reglamentación de las nanotecnologías.

En 2005, fue aprobada en México ${ }^{8}$ una exhortación para la elaboración de un programa de emergencia en nanotecnología por la Comisión de Ciencia y Tecnología del Senado. 9 A finales de 2013, una propuesta en la misma comisión exhorta a la elaboración de un programa de desarrollo y su regulación, por la necesidad de acelerar su desarrollo, habida cuenta de los potenciales riesgos a la salud humana y el medio ambiente de las nanopartículas manufacturadas o nanoestructuras,$^{10}$ aún en trámite.

Pero México ha dado pasos más significativos. Ha sido el primero en elaborar unos lineamientos voluntarios para la regulación de las nanotecnologías a instancias del gobierno de Estados Unidos, prácticamente reproduciendo sin modificación los lineamientos del país del norte." El documento mexicano, elaborado por una comisión coordinada

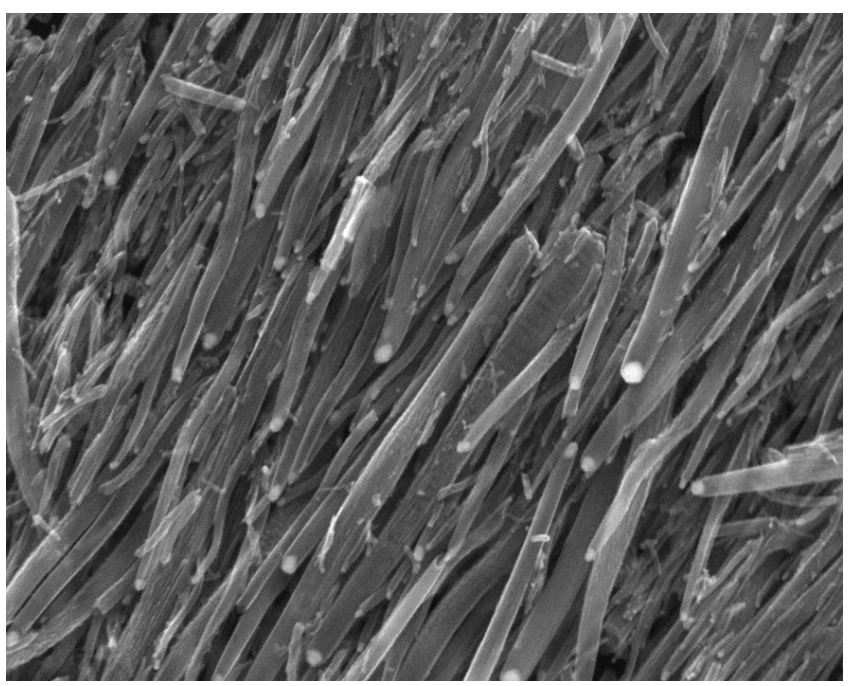

por el Centro de Metrología de la Secretaría de Economía consiste en i2 puntos y está básicamente orientado a facilitar el tráfico comercial antes que en salvaguardar la salud humana y el medio ambiente. El documento escapa de un enfoque precautorio y tiende a que cada caso sea negociado de manera independiente, enlenteciendo de esa manera cualquier tipo de regulación y ajustándose a lo que el documento madre de Estados Unidos indica.

En Brasil, ${ }^{12}$ los intentos por comenzar la reglamentación datan de 2005. El primero de ellos, de 2005, es obra del diputado federal Edson Duarte (Partido Verde - Pv-) quien presentó el proyecto de ley $\mathrm{N}^{0} 5.076 / 2005$ con el cual se pretendía crear la Comisión Técnica Nacional de Nanoseguridad y el Fondo de Desarrollo de Nanotecnología. En el proyecto se pretendía demostrar que la nanotecnología implica riesgos para la salud y el medio ambiente, y que es necesario que el consumidor esté informado sobre los productos con nanotecnología y se dé un control de seguridad al tema. El proyecto fue desechado.

En 2010 se presentó un proyecto para el etiquetado de alimentos, medicinas y otros productos sujetos al régimen de vigilancia sanitaria. Este proyecto de ley, el I3I/20ıo, quería asegurar el derecho de información al consumidor sobre los productos de la nanotecnología. El proyecto también fue desestimado.

En marzo y noviembre de 2013 se presentaron los proyectos de ley $5.133 / 2013$ y $6.74 \mathrm{I} / 2013$. Ambos presentados por el diputado federal Sarney Filho (PV). En el primero se busca etiquetar todos los productos a base de nanotecnología, y los que se utilizan en cosméticos, alimentos y fármacos; también se 


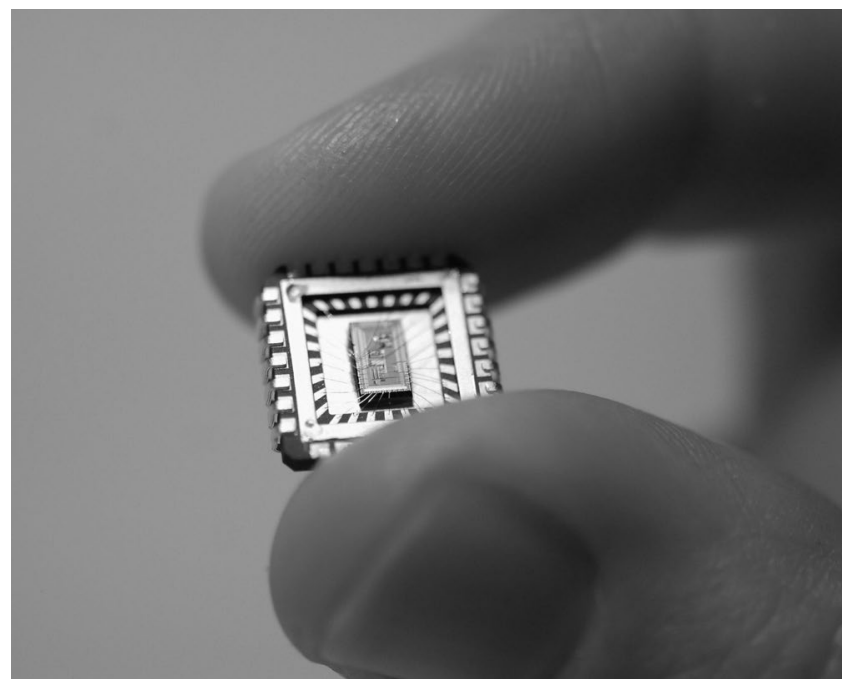

busca incluir en el etiquetado la nanomateria prima utilizada. El segundo pretende instaurar una Política Nacional de Nanotecnología con foco en el incentivo a la investigación, desarrollo tecnológico y control por el poder público de los riesgos e impactos, ambos proyectos de ley se encuentran actualmente (agosto de 20I4) en estudio por la Cámara de Diputados para su estudio y aprobación.

Paralelamente, la Comisión Interministerial de Nanotecnología tiene en su agenda la discusión de una reglamentación, aunque aún no se han dado pasos firmes en esto. El resto de los países de América Latina no tienen avances significativos en el tema.

\section{- notas $\cdot-$}

' Este apartado sobre el SAICM fue proporcionado por Guillermo Foladori.

' UNEP (2013, February 5), Outcomes of the Third International Conference on Chemicals Management (ICCM3) I7-2I September 2012, Nairobi, Kenya. United Nations Environment Programme.

${ }^{3}$ Bergeson, L. (2012). ICCM3 Adds Measures Concerning Nanotechnologies And Manufactured Nanomaterials To Global Plan Of Action : Nano and Other Emerging Technologies Blog: ICCM SAICM "Third International Conference on Chemicals Management" "Strategic Approach to International Chemicals Management" GHS UN "United Nations." http:/nanotech.lawbc. com/2012/Io/articles/international/iccm3-adds-measures-concerning-nanotechnologies-and-manufactured-nanomaterialsto-global-plan-of-action/print.html.

${ }^{4}$ Comisión de las Comunidades Europeas. (2004, May 12). Hacia una estrategia europea para las nanotecnologías. Comisión de la Comunidad $\operatorname{COM}(2004) 338$ final. http://eur-lex.europa.eu/legal-content/ES/TXT/PDF/?uri=CELEX:52004DCo338\&from=ES.

${ }^{5}$ Commission Recommendation. (20II). Commission Recommendation of 18 October 201 on the definition of nanomaterial. Official Journal of the European Union, L 275/38. http://eurlex.europa.eu/LexUriServ/LexUriServ.do?uri=OJ:L:20п:275:003 8:0040:EN:PDF.

${ }^{6}$ EPA. (n.d.). Making a Finding on Unreasonableness of Risk. Retrieved August 25, 20I4, from http:/www.epa.gov/oppt/ newchems/pubs/unrerisk.htm.

7 Holdren, J. P., Sustain, C. R., \& Siddiqui, I. A. (20II, June 9). Policy Principles for the U.S. Decision-Making Concerning
Regulation and Oversight of Applications of Nanotechnology and Nanomaterials. Memorandum for the heads of executive departments and agencies. http://www.whitehouse.gov/sites/ default/files/omb/inforeg/for-agencies/nanotechnology-regulation-and-oversight-principles.pdf.

${ }^{8}$ Los siguientes párrafos sobre México fueron resumidos de Foladori, G., \& Záyago Lau, E. (2014). The regulation of nanotechnology in Mexico. Nanotechnology Law \& Business, 11(2), І64-17г.

9 Alanís Quiñones. (n.d.). Senado de la República [Comisión de Ciencia y Tecnología Senado]. http://www.senado.gob. $\mathrm{mx} /$ ? ver $=$ sp\&mn=2\&sm=2\&id=7529.

${ }^{10}$ Robles-Montoya, A. (2013). Proposición al Senado de la República relativo al diseño de un progrma de investigación y desarrollo de la nano ciencia y la nanotecnología. Senado de La República. LXLI Legislatura Del Congreso de La Unión. México. http://www.senado.gob.mx/?ver=sp\&mn=2\&sm=2\&id=4387г

"Grupo de trabajo sobre regulaciones para la nanotecnología. (2012, November 26). Lineamientos para regulaciones sobre nanotecnologías para impulsar la competitividad y proteger al medio ambiente, la salud y la seguridad de los consumidores. Secretaría de Economía. http:/www.economia.gob.mx/files/comunidad_negocios/normalizacion/dgn/20I2_II_27_Lineamientos_regulaciones_nanotecnologia.pdf.

${ }^{12}$ Los párrafos que siguen sobre Brasil fueron tomados de Engelmann, Primeras tentativas de reglamentación de las nanotecnologías en Brasil, de próxima aparición en el libro de Foladori et al, Trabajo, Regulación y Nanotecnologías en América Latina, Miguel Ángel Porrúa, México 2015. 